\title{
Engaging the p53 metabolic brake drives senescence
}

\author{
Cell Research (2013) 23:739-740. doi:10.1038/cr.2013.34; published online 12 March 2013
}

Emerging evidence suggests that the ability of p53 to regulate metabolism is important for its tumor suppressor activity. A recent study published in Nature reveals a novel connection between $p 53$ and metabolism: p53 transcriptionally represses the expression of malic enzymes and associated NADPH production, which in turn triggers a positive feedback loop resulting in sustained p53 activation, cellular senescence, and tumor suppression.

The p53 tumor suppressor plays a key role in combatting malignant transformation [1]. p53 is a cellular stress sensor that induces cell cycle arrest, cellular senescence or apoptosis in response to stresses such as DNA damage and oncogenic signals. p53 drives these responses primarily through its ability to regulate networks of downstream target genes. While years of $\mathrm{p} 53$ research have focused on elucidating the molecular details of how p53 triggers cell cycle arrest or apoptosis in response to acute DNA damage, it has become clear from recent in vivo studies in mouse models that these responses are dispensable for tumor suppression [2-3], raising the question of which p53 functions are of paramount importance for suppressing cancer development. Interestingly, increasing evidence over the past decade has illuminated an active role for $\mathrm{p} 53$ in the regulation of cellular metabolism, echoing the recognition of metabolic reprogramming as a new hallmark of cancer [4-5]. Indeed, a diversity of studies has revealed that $\mathrm{p} 53$ counteracts cancer-associated metabolic transformation at various points in the metabolic network. Underscoring the importance of this function for tumor suppression, a recent study in mouse models has suggested that the ability of p53 to regulate metabolism and to promote antioxidant responses is intimately associated with tumor suppression [6]. These observations emphasize the critical importance of further elaborating the role of p53 in regulating metabolism and how this contributes to cancer suppression.

One of the hallmarks of tumor cells is that they actively engage aerobic glycolysis as a means to generate components for macromolecular synthesis and for NADPH production [7]. NADPH is critical for anabolic metabolism, as it provides reducing equivalents necessary for lipid biosynthesis, and facilitates the production of reduced glutathione, an important antioxidant, to protect tumor cells from reactive oxygen species (ROS)-induced damage. Inhibiting NADPH production may therefore represent an effective strategy to shut down tumor growth. Previous studies from the Yang group have revealed that p53 is implicated in restricting NADPH production by inhibiting glucose flux through the pentose phosphate pathway (PPP) through its direct binding to glucose-6-phosphate dehydrogenase (G6PD), the rate-limiting enzyme of the PPP [8]. Now, Jiang et al. [9] unveil another mechanism by which p53 suppresses NADPH production to impede tumorigenesis.

Reasoning that malic enzymes (MEs) catalyze the conversion of malate into pyruvate while concomitantly producing NADPH, the authors investigated the link between p53 and the malic enzymes ME1 and ME2. They found that p53 accumulation resulted in di- minished ME protein levels, an effect mediated through direct binding of p53 to the response elements in the introns of the $M E 1$ and $M E 2$ genes and subsequent transcriptional repression. Using RNA interference and overexpression approaches, the authors showed that the MEs, particularly ME2, are required for optimal NADPH production, lipid synthesis and glutaminolysis, and that p53 negatively regulates these metabolic processes through ME2 repression. Intriguingly, upon ME1 or ME2 silencing, the authors noted a robust p53-dependent proliferative arrest, associated with markers of senescence and the induction of multiple senescenceassociated p53 target genes. Consistent with an important role in regulating senescence, ME2 levels declined during replicative senescence in human fibroblasts, and enforced expression of either ME1 or ME2 delayed replicative senescence in a manner dependent on ME enzymatic activity. When further querying the connection between MEs and p53-dependent senescence, Jiang et al. [9] discovered that p53 levels were increased by ME knockdown and decreased by ME overexpression, and they elaborated mechanistically distinct pathways through which ME1 and ME2 regulate $\mathrm{p} 53$ levels. ME1 silencing caused reduced expression of MDM2, a negative regulator of p53 stability and activity. ME2 knockdown, on the other hand, led to increased cellular ROS levels, which provoked AMP-activated protein kinase (AMPK) to promote p53 phosphorylation and activation. Thus, these findings identify a positive feedback loop whereby p53 represses MEs, leading to additional stabilization 


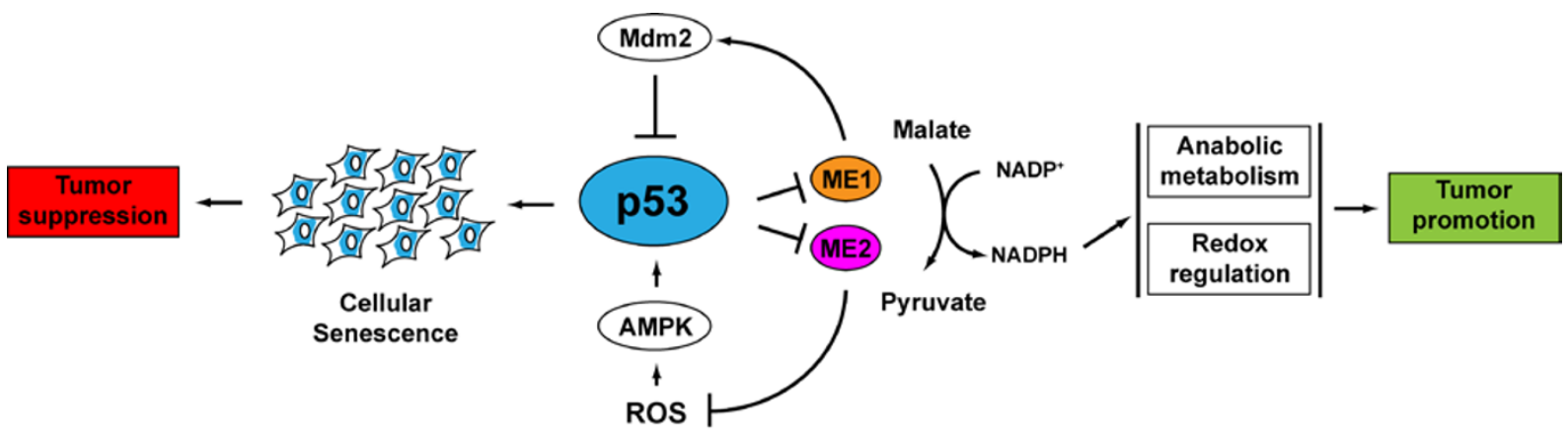

Figure 1 Reciprocal regulation between p53 and malic enzymes. p53 directly represses transcription of ME1 and ME2, which encode enzymes catalyzing the conversion of malate to pyruvate, with concomitant generation of NADPH. NADPH is essential for anabolic metabolism and cellular antioxidant function, both of which are implicated in tumor promotion. Suppression of ME1 or ME2 expression induces p53 activation, cellular senescence and tumor suppression through MDM2 and ROS/AMPK pathways, respectively.

of $\mathrm{p} 53$, which enhances its function in senescence. Importantly, ME1 or ME2 knockdown impeded malignant transformation and tumor growth in both in vitro soft agar assays and in vivo xenograft assays, underscoring the functional importance of the metabolic brake imposed by $\mathrm{p} 53$ for cancer suppression.

The study from Jiang et al. [9] has uncovered a new facet of $\mathrm{p} 53$ function in metabolic regulation, highlighting a more profound role of $\mathrm{p} 53$ in the surveillance of cellular NADPH levels (Figure 1). Notably, an earlier study showed that p53 can drive glucose flux through the PPP by regulating another target gene TIGAR- enhancing NADPH production and protecting cells from ROS-induced apoptosis [10]. This apparently opposite role for p53 in regulating metabolism emphasizes the complexity of metabolic regulation, where context may dictate the exact wiring of pathways. The reciprocal regulation between $\mathrm{p} 53$ and ME1/2 highlights the bidirectional interplay between p53 and metabolic pathways. Accordingly, p53 has been found not only to regulate metabolic pathways but also to be activated by metabolic stresses such as nutrient deprivation and hypoxia. The work presented by Jiang et al. [9] suggests that metabolic changes can provoke p53 to induce cellular senescence, and thus prevent tumorigenesis. Future investigations will continue to unravel the intricacies of how p53 regulates metabolism to suppress cancer development.

\section{Dadi Jiang ${ }^{1}$, Laura D Attardi ${ }^{1,2}$}

${ }^{1}$ Division of Radiation and Cancer Biology, Department of Radiation Oncology, ${ }^{2}$ Department of Genetics, Stanford University School of Medicine, Stanford, CA 94305, USA
Correspondence: Laura D Attardi

E-mail: attardi@stanford.edu

\section{References}

1 Vousden KH, Prives C. Cell 2009; 137:413431.

2 Christophorou MA, Ringshausen I, Finch AJ, et al. Nature 2006; 443:214-217.

3 Brady CA, Jiang D, Mello SS, et al. Cell 2011; 145:571-583.

4 Hanahan D, Weinberg RA. Cell 2011; 144:646-674.

5 Maddocks OD, Vousden KH. J Mol Med (Berl) 2011; 89:237-245.

6 Li T, Kon N, Jiang L, et al. Cell 2012; 149:1269-1283.

7 Vander Heiden MG, Cantley LC, Thompson CB. Science 2009; 324:1029-1033.

8 Jiang P, Du W, Wang X, et al. Nat Cell Biol 2011; 13:310-316.

9 Jiang P, Du W, Mancuso A, et al. Nature 2013; 493:689-693.

10 Bensaad K, Tsuruta A, Selak MA, et al. Cell 2006; 126:107-120. 